\title{
TECHNOLOGY WATCH
}

\section{A small world gets bigger}

MicroRNAs (miRNAs) are 22 -nucleotide non-coding RNA molecules that inhibit the translation or induce the degradation of protein-coding mRNAs in plants and animals. Recently, a member of the human herpesvirus family - Epstein-Barr virus - was found to encode miRNAs. These miRNAs do not have close homologues in other viral genomes or in the genome of their host, so it is difficult to identify other viral miRNAs using the available prediction software. Now, in Nature Methods, Tuschl and colleagues describe the development of a new computational method to identify miRNAs.

Their method predicts the probable location of miRNA precursors in a genome using information about local sequence composition and the predicted secondary structure of RNA transcripts (it assumes that miRNA precursors have specific sequence and structural features that are recognized by the processing enzymes). They applied this method to a subset of pathogenic DNA and RNA viruses (herpesviruses and smallgenome RNA viruses, respectively) and, when possible, using their results as a guide, they cloned and sequenced small RNAs from virus-infected cells. As a result, they confirmed their prediction of many miRNAs in herpesviruses and, consistent with low prediction scores, they could not experimentally identify miRNAs in small-genome RNA viruses. This technique is therefore a good starting point for the identification of viral miRNAs.

REFERENCE Pfeffer, S. et al. Identification of microRNAs of the herpesvirus family. Nature Meth. 16 Feb 2005 (doi:10.1038/nmeth746) Cell the characterization of Shifted (Shf), a secreted protein that is required to control the movement of Hh in Drosophila melanogaster.

Both groups analysed the function of shfin Hh signalling in the developing wing as a model system. Known mutations in shfcaused defects that were similar to those of mutants that are known to affect the release, transport or modification of Hh. Both groups found that the levels of Hh protein were reduced, as was the range of Hh movement and the resultant signalling. This indicated that Shf was required for both the accumulation or stability of $\mathrm{Hh}$ and its movement within the wing disc from the posterior compartment (Hh-producing cells) to the anterior compartment (Hh-receiving cells).

Further characterization of the shf locus by both groups showed it to encode the D. melanogaster orthologue of the vertebrate Wnt inhibitory factor-1 (WIF-1). Both proteins have an N-terminal signal sequence, a WIF domain and five epidermal growth factor (EGF)-like repeats. In addition to the presence of the signal sequence - an indicator of secretion - non-autonomous rescue

experiments and immunofluorescence indicated that Shf was secreted.

Both groups also observed a relatively higher level of Shf in cells of the posterior compartment, which depended on the presence of Hh. And Gorfinkiel et al. were able to co-immunoprecipitate Shf and Hh. Both sets of authors surmised that Shf might mediate the interaction between $\mathrm{Hh}$ and heparan sulphate proteoglycans (HSPGs), which, like Shf, are necessary for the normal accumulation and movement of $\mathrm{Hh}$. And, indeed, Glise et al. showed that changes in HSPGs affected Shf accumulation (as well as Hh distribution).

In vertebrates, WIF-1 inhibits Wnt signalling, but both groups agreed that Shf modulates the distribution of Hh without affecting Wnt signalling. Gorfinkiel et al. further showed that human WIF inhibited signalling by the Wnt-family member Wingless in D. melanogaster without affecting the Hh pathway. So different WIF-family members might have divergent functions in each pathway.

Katrin Bussell

\section{(1) References and links} ORIGINAL RESEARCH PAPERS Gorfinkiel, N. et al. The Drosophila ortholog of the human Wnt inhibitory factor Shifted controls the diffusion of lipid-modified Hedgehog. Dev. Cell 8, 241-253 (2005) | Glise, B. et al. Shifted, the Drosophila ortholog of Wnt inhibitory factor-1, controls the distribution and movement of Hedgehog. Dev. Cell 8, 255-266 (2005)

FURTHER READING Hooper, J. E. \& Scott, M. P. Communicating with Hedgehogs. Nature Rev. Mol. Cell Biol. 6, 306-317 (2005)

\section{A restricted reaction}

Single-molecule techniques have given us insights into molecular behaviours that often go unobserved in multiple-molecule experiments. With respect to enzymatic studies, single-molecule techniques can be used to monitor conformational changes in enzymes or binding/release events, but they are blind to the products of enzymatic reactions. One way to allow the products to be considered would be to inhibit their diffusion by carrying out the experiments in very small containers, and Noji and coworkers now describe suitable containers in Nature Biotechnology. They used poly-dimethylsiloxane (PDMS), a silicone elastomer, to build a device that contains a large array of micrometre-sized cavities. By enclosing a liquid solution between this micropatterned sheet and a glass microscope slide, they could form femtolitre water volumes that were stable over long periods of time. PDMS is sufficiently impermeable to prevent evaporation or leakage for the duration of a typical bioassay, and the sheet ensures that the chambers have a uniform size. The authors proved that their device can be used to monitor the individual activities of isolated single enzymes — in particular, to monitor reaction products - by studying $\beta$-galactosidase and horseradish peroxidase as models. When trapped in a femtolitre chamber, a single molecule of each of these enzymes only needed a few turnovers to accumulate detectable concentrations of their products. This technique should therefore be useful for "...many ultrasensitive bioassays at the single-molecule level".

REFERENCE Rondelez, Y. et al. Microfabricated arrays of femtoliter chambers allow single molecule enzymology. Nature Biotechnol. 20 Feb 2005 (doi:10.1038/nbt1072) 